\title{
A High Fuel Consumption Efficiency Management Scheme for PHEVs Using an Adaptive Genetic Algorithm
}

\author{
Wah Ching Lee ${ }^{1}$, Kim Fung Tsang ${ }^{2, *}$, Hao Ran Chi ${ }^{2}$, Faan Hei Hung ${ }^{2}$, Chung Kit Wu ${ }^{2}$, \\ Kwok Tai Chui ${ }^{2}$, Wing Hong Lau ${ }^{2}$ and Yat Wah Leung ${ }^{3}$ \\ 1 Department of Electronic and Information Engineering, Hong Kong Polytechnic University, \\ Hung Hom, Kowloon, Hong Kong, China; E-Mail: enwclee@polyu.edu.hk \\ 2 Department of Electronic Engineering, City University of Hong Kong, Kowloon Tong, Kowloon, \\ Hong Kong, China; E-Mails: haoranchi2-c@cityu.edu.hk (H.R.C.); fhhung4@cityu.edu.hk (F.H.H.); \\ chungkwu4@gapps.cityu.edu.hk (C.K.W.); ktchui3-c@my.cityu.edu.hk (K.T.C.); \\ itwhlau@cityu.edu.hk (W.H.L.) \\ 3 Department of Systems Engineering and Engineering Management, Hong Kong Polytechnic \\ University, Hung Hom, Kowloon, Hong Kong, China; E-Mail: 50997159@student.cityu.edu.hk \\ * Author to whom correspondence should be addressed; E-Mail: ee330015@cityu.edu.hk; \\ Tel.: +852-3442-7806; Fax: +852-3442-0562.
}

Academic Editor: Vittorio Passaro

Received: 20 October 2014 / Accepted: 7 January 2015 / Published: 12 January 2015

\begin{abstract}
A high fuel efficiency management scheme for plug-in hybrid electric vehicles (PHEVs) has been developed. In order to achieve fuel consumption reduction, an adaptive genetic algorithm scheme has been designed to adaptively manage the energy resource usage. The objective function of the genetic algorithm is implemented by designing a fuzzy logic controller which closely monitors and resembles the driving conditions and environment of PHEVs, thus trading off between petrol versus electricity for optimal driving efficiency. Comparison between calculated results and publicized data shows that the achieved efficiency of the fuzzified genetic algorithm is better by $10 \%$ than existing schemes. The developed scheme, if fully adopted, would help reduce over 600 tons of $\mathrm{CO}_{2}$ emissions worldwide every day.
\end{abstract}

Keywords: PHEV; adaptive genetic algorithm; fuel efficiency management 


\section{Introduction}

Global warming has gradually become a big issue and it is aggravating because of increasing $\mathrm{CO}_{2}$ emissions [1]. In order to minimize $\mathrm{CO}_{2}$ and potential pollutants generated by burning fuels, much cleaner alternative energy sources for the next generation of vehicles are desperately sought for [2,3]. Hybrid Electric Vehicles (HEVs) are new cars manufactured for the purpose of generating little or minimum pollutants. A higher HEV fuel efficiency will help to alleviate the carbon release issue. In HEVs, fuel and electric power are available energy sources and may provide energy at the same time [4]. The fuel efficiency of HEV, $\eta_{\mathrm{H}}$, is defined as the percentage of energy consumption due to fuel combustion among the total energy consumed. In particular, in plug-in Hybrid Electric Vehicles (PHEVs), the internal combustion engine is connected directly to the wheels in parallel to the electric motor. Thus, the fuel efficiency of PHEV, $\eta \mathrm{P}$, in general surpasses the respective $\eta_{\mathrm{H}}$. Higher efficiency $(\eta)$ implies less contamination, rendering a greener environment. A careful review of the existing fuel efficiency schemes reveals that there is still room for improvement of $\eta p$. Intuitively, if the driving control mechanism conforms to the road conditions, a higher $\eta p$ will be achieved. In existing PHEV energy consumption schemes, it is observed that under the circumstance of fast speed and high acceleration, more pollutants will be produced than during uniform driving because more fuel is consumed. Thus a more efficient scheme to trade-off fuel-versus-electricity (FVE) is needed. In this communication, an efficient optimization algorithm for PHEV that pertains to road and driving conditions has been developed to achieve the FVE target.

Genetic algorithm (GA) is a good candidate for global optimization [5,6] and former optimizations of $\eta_{P}$ were attempted [7-9]. It is noted that in former works, vital parameters of PHEVs such as the battery State of Charge (SOC) and the fuel capacity (C), were not considered as key factors in the optimization of $\eta_{\mathrm{P}}$. In this new scheme, the choice of resource consumption between fuel and electricity must take into account the road (e.g., uphill, downhill etc.) effect and car condition (e.g., high battery level and/or low fuel level etc.). The status of SOC and C thus are indicative of the road and car conditions. In reality, clearcut boundaries must be devised to help decision making for choice of resources. The existing scheme incorporates a fuzzy logic controller (FLC) which fuzzifies the inputs, namely SOC and C. The fuzzification will moderate the objective function of GA. This process is referred as Fuzzified GA (FGA) which is the key contribution in this investigation. The output from the FGA will then drive the PHEV. Such a process will ensure the best $\eta_{P}$ that pertains to the ever changing road conditions. The fuzzified GA scheme (FGAS) will then trade off FVE. By virtue of the characteristics of FGAS, a plurality of stable objective functions is achieved, rendering a high $\eta$ p. It will be shown from simulated results that FGAS is more efficient than the traditional GA scheme (TGAS).

\section{Design of TGAS for PHEV}

Basic vital parameters that may affect significantly the efficiency of a traditional GAS are listed in Table 1. Settings of the population size and maximum generations conform to the Allele Coverage [10,11]. Such a setting is representative in the design of TGAS.

It was explained that both $\mathrm{C}$ and SOC are key parameters of PHEVs. In Table 1, in the ten bits of individuals, the first five binary bits represent the current SOC and the last five bits indicate the current 
percentage of $\mathrm{C}$. The "SOC" and "C" status divide the whole range $[0 \%, 100 \%]$ into thirty-two stages, with intervals of less than $4 \%$, which is sufficient to represent the state of SOC and C accurately.

Table 1. GA (FGA) parameters of population and selection.

\begin{tabular}{cc}
\hline GA (FGA) Parameters & Designed Data of GA (FGA) Parameters \\
\hline Population size & 100 \\
Number of maximum generations & 100 \\
Number of bits of individuals & 10 \\
Selection method & Roulette Wheel Selection (RWS) \\
\hline
\end{tabular}

Uniform crossover with a rate of 0.6 and mutation with a rate of 0.01 are adopted as the main genetic operations based on natural experience [12-14]. Objective functions are vital elements of the TGAS. The objective function, $F_{x}$, is shown in Equation (1).

$$
\text { MAX: } F_{x}=\sum_{i=1}^{10} b_{i} f(i)
$$

where $F_{x}$ is the fitness value of the objective function for the individual $x, b_{i}$ is the binary bit $i$ of the individual, $f(i)$ is the value of $b_{i}$ and $f(i)$ is designed as:

$$
f(i)=\left\{\begin{array}{c}
7-i, i \in[1,5] \\
i-11, i \in[6,10]
\end{array}\right.
$$

The output $F_{x}$ of TGAS represents $\eta \mathrm{P}$, which is calculated as the ratio of consumed electric power to the total energy consumption.

\section{Design of FGAS for PHEV}

The working principle of FGAS is shown in Figure 1. The initialization values of GA and FGA are the same and are shown in Table 1. It was explained in earlier context that SOC and C from PHEV are representative of road conditions. At time $t$, the PHEV provides output PHEV feedback variables, $S_{\mathrm{PHEV}, t}$ and $\mathrm{C}_{\mathrm{PHEV}, t}$, to the GA. The output $\mathrm{GA}$ variable $1, \mathrm{SOC}_{t}$ and $\mathrm{C}_{t}$, at the output of the GA are then fuzzified by the FLC. The output fuzzified variable, $y_{t}$, moderates the GA variables, SOC $_{t}$ and $C_{t}$, to yield an adaptive $\eta_{\mathrm{p}, t+1}$. The $\eta_{\mathrm{p}, t+1}$ will trade off fuel versus electricity for PHEV steering and generate $\mathrm{SOC}_{\mathrm{PHEV}, t+1}$ and $C_{\mathrm{PHEV}, t+1}$ which in turn generate $\mathrm{SOC}_{t+1}$ and $\mathrm{C}_{t+1}$. The feedback control process reiterates until the optimal $\eta_{P}$ is achieved.

\section{Design of FLC}

The fuzzy input variables of FLC, SOC and C, are shown in Table 2. The fuzzification design is categorized into fuzzy sets ranging from "Very low" to "very high" respectively in five levels $\left(\mathrm{SOC}_{1}-\mathrm{SOC}_{5}, \mathrm{C}_{1}-\mathrm{C}_{5}\right)$. The design of fuzzy sets of input FLC variables is shown in Table 2. 


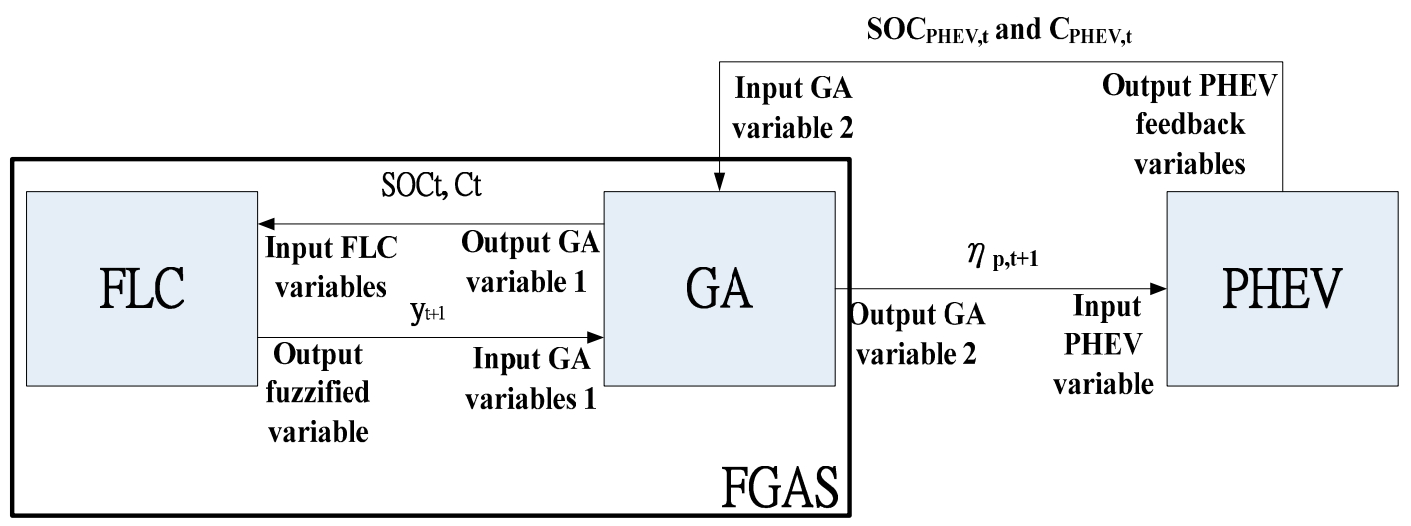

Figure 1. Fuzzified GA scheme (FGAS).

Table 2. Design of fuzzy sets of inputs of FLC.

\begin{tabular}{|c|c|c|}
\hline Inputs & Function Types & $\begin{array}{l}\text { Designs (L\&U: Lower and Upper Bounds, C: Centre, } \\
\text { W: Width of the Top Side of a Trapezoidal Function) }\end{array}$ \\
\hline \multirow{2}{*}{ SOC } & & $(\mathrm{L}, \mathrm{U}, \mathrm{C}, \mathrm{W})=(-0.2,0.35,0.15,0.15),(0.75,1.2,0.95,0.05)$ \\
\hline & Triangular: $\mathrm{SOC}_{2}, \mathrm{SOC}_{3}, \mathrm{SOC}_{4}$ & $(\mathrm{~L}, \mathrm{C}, \mathrm{U})=(0.15,0.35,0.55),(0.35,0.55,0.75),(0.55,0.75,0.95)$ \\
\hline & Trapezoidal: $\mathrm{C}_{1}, \mathrm{C}_{5}$ & $(\mathrm{~L}, \mathrm{U}, \mathrm{C}, \mathrm{W})=(-0.1,0.45,0.25,0.25),(0.85,1.3,1.05,0.15)$ \\
\hline & Triangular: $\mathrm{C}_{2}, \mathrm{C}_{3}, \mathrm{C}_{4}$ & $(\mathrm{~L}, \mathrm{C}, \mathrm{U})=(0.25,0.45,0.65),(0.45,0.65,0.85),(0.65,0.85,1.05)$ \\
\hline
\end{tabular}

The design in Table 2 renders the SOC always has a larger value than $\mathrm{C}$. In the design, the range of $\operatorname{SOC}_{x}(x=1,2, \ldots 5)$ is slightly smaller than $\mathrm{C}_{x}(x=1,2, \ldots 5)$. For instance, when $80 \%$ SOC is classified as "very high" $\left(\mathrm{SOC}_{5}\right)$, the fuel capacity $\left(\mathrm{C}_{4}\right)$ will likely be just "high". Thus it is seen that with identical proportion of SOC and $\mathrm{C}, \mathrm{SOC}$ is a stronger indicator than $\mathrm{C}$.

As shown in Table 2, there are twenty five fuzzy rules designed. The format is designed as IF THEN rules, which is shown as "IF SOC is $\mathrm{SOC}_{x}$ and $\mathrm{C}$ is $\mathrm{C}_{x}$, THEN the output FLC variable is $\mathrm{Y}_{x}$ ", where $Y_{x}$ are the fuzzy sets at the output. Finally, the deffuzified output at the moment $t, y_{t}$, is calculated by using the centre of area method [9] and given by Equation (3):

$$
y_{t}=\frac{\sum_{x=1}^{n} \alpha_{x}(t) \mathrm{Y}_{x}}{\sum_{x=1}^{n} \alpha_{x}(t)}
$$

where $\alpha_{x}(t)$ is strength of the output belonging to the fuzzy sets $\mathrm{Y}_{x}$ at time $\mathrm{t}(x=1,2, \ldots, 5)$.

The fuzzified output FLC variable, $y_{t}$, will then become the input GA variable 1. Attention is drawn to the point that the GA functions of FGAS and GAS are different. In FGAS, the GA function is given by Equation (4):

$$
f(i)=\left\{\begin{array}{c}
y_{t}(7-i), i \in[1,5] \\
y_{t}(i-11), i \in[6,10]
\end{array}\right.
$$

\section{Simulated Results and Discussion}

The design is mainly based on the practical facts of private vehicles in Hong Kong. Under the uniform driving condition, various initial values of SOC and C are used for simulation. The objective is to investigate if FGAS is more adaptive and efficient than TGA in improving $\eta$ of PHEVs. TOYOTA 
PRIUS, a popular PHEV in the market, is chosen as a benchmark. Detailed design of the scenarios is described in Table 3.

Table 3. Scenario settings for the comparison of FGAS versus TGAS.

\begin{tabular}{cc}
\hline Parameters & Designed Data \\
\hline Driving condition & Uniform driving \\
Average speed & $50 \mathrm{~km} / \mathrm{h}$ \\
SOC $(\%)$ & $100-10 \mathrm{k}(\mathrm{k}=0,1,2, \ldots, 9)$ \\
C $(\%)$ & $100-10 \mathrm{~m}(\mathrm{~m}=0,1,2, \ldots, 9)$ \\
Max. capacity of fuel tank & $36 \mathrm{Litr}$ \\
Max. electric power & $45 \mathrm{~kW}$ \\
\hline
\end{tabular}

In the design, 10 states of both SOC and $\mathrm{C}$ are used. There will be in total 100 scenarios, which are sufficient to elaborate the practical states of PHEVs. During a $50 \mathrm{~km}$ drive in a fluctuating environment, initial values of both SOC and C deviate from the initial state of PHEVs by varying the charge of the battery and fuel level in the fuel tank. It is expected that with an adaptive scheme, a higher accurate and more efficient performance can be achieved. The simulated results of all the 100 scenarios are shown in Figure 2. The average improvement of GA is $8.7 \%$. However, FGA can achieve an improvement of about $18.9 \%$. With unchanged objective functions of TGA, the performance is similar with FGA in those scenarios with high SOC and C. However, in most other scenarios, the premature convergence of TGA occurs frequently. Alternatively, FGA can adjust the objective functions by the fuzzy control action pertaining to the environment to provide a better performance. Therefore, higher fuel efficiency can be achieved by FGA.

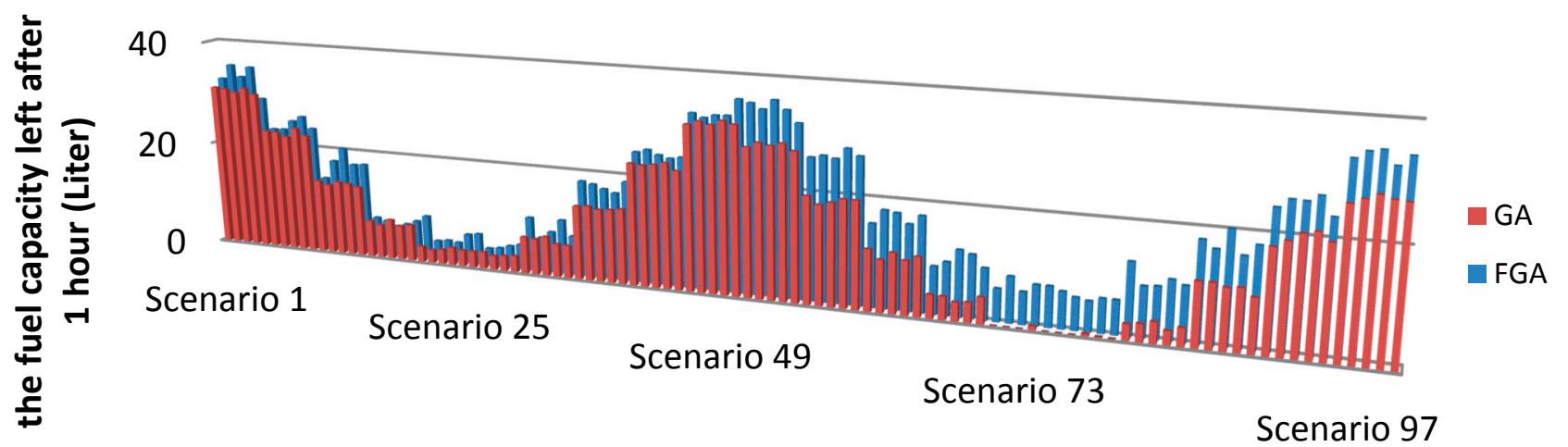

Scenario number

Figure 2. Fuel capacity after one hour (100 scenarios in total).

As an illustration, the cumulative sales volume of PHEV is more than 7 million units, among which there are more than 4.2 million current users [15]. Based on these figures and from TOYOTA PRIUS-C [16], the average distance that one PHEV travels per day is $28 \mathrm{~km}$ which is equivalent to $1 \mathrm{~L}$ fuel consumption. It is evidenced that $1 \mathrm{~L}$ fuel produces $2.2 \mathrm{~kg} \mathrm{CO}_{2}$ [17] and that the improvement of FGAS is $10 \%$. As a result, more than $186,880 \mathrm{~L}$ of fuel can be saved. This represents a reduction of emissions of $\mathrm{CO}_{2}$ by 613 tons every day. 


\section{Conclusions}

A fuzzified genetic algorithm (FGA) has been developed to deal with the fuel efficiency management of PHEVs. In FGA, a fuzzy logic controller has been designed to provide control input to the objective functions of genetic algorithm (GA) scheme to ensure FGA be adaptive to the ever changing environment. As a result, the FGA is more efficient than traditional GA (TGA) schemes under unchanged objective functions. Simulated results indicate that FGA has an average improvement of $18.9 \%$ while TGA achieves an improvement of $8.7 \%$. Therefore the efficiency of the FGA is $10 \%$ better than TGA. With such an improvement, FGA can reduce the $\mathrm{CO}_{2}$ emissions by over 600 tons worldwide every day if all PHEVs were to adopt FGA, hence FGAS is a more efficient scheme for the fuel efficiency management of PHEVs.

\section{Acknowledgments}

This work was financially supported by the Department of Electronic Engineering, City University of Hong Kong through project GRF116013 from UGC of the government of the Hong Kong SAR, China. The support from the Wireless Sustainability Center of the Department of Electronic Engineering, City University of Hong Kong is gratefully acknowledged.

\section{Author Contributions}

W.C. Lee has coordinated the theoretical and experimental developments, participating in the establishment of the theory principles used in this work, as well as in the drafting of the manuscript. K.F. Tsang and H.R. Chi have developed part of the theory used and have designed some characterization experiments, as well as have participated in the drafting of the manuscript. F.H. Hung, C.K. Wu and K.T. Chui have designed and carried out the characterization of the experimental setup and in computing algorithms for the treatment of data, also have participated in the analysis of the state of art and in the design of some experiments, as well as in the drafting of the manuscript. W.H. Lau and D. Leung have developed some computing task, and have participated in the design of some experiments, as well as in the drafting of the manuscript.

\section{Conflicts of Interest}

The authors declare no conflict of interest.

\section{References}

1. Geórgia, M.; Maria, C.; Marcelo, S.; Guilherme, L.; Laisa, B.; Layse, C.; Aline, R.; Helion, V. Detection of Greenhouse Gas Precursors from Diesel Engines Using Electrochemical and Photoacoustic Sensors. Sensors 2010, 10, 9726-9741.

2. Schlote, A.; Hausler, F.; Hecker, T.; Bergmann, A.; Crisostomi, E.; Radusch, I.; Shorten, R. Cooperative Regulation and Trading of Emissions Using Plug-in Hybrid Vehicles. IEEE Trans. Intell. Transp. Syst. 2013, 14, 1572-1585. 
3. Bucak, I.O. Position Error Compensation via a Variable Reluctance Sensor Applied to a Hybrid Vehicle Electric Machine. Sensors 2010, 10, 1918-1934.

4. Onar, O.C.; Kobayashi, J.; Erb, D.C.; Khaligh, A. A Bidirectional High-Power-Quality Grid Interface with a Novel Bidirectional Noninverted Buck-Boost Converter for PHEVs. IEEE Trans. Veh. Technol. 2012, 61, 2018-2032.

5. Balieiro, A.; Yoshioka, P.; Dias, K.; Cordeiro, C.; Cavalcanti, D. Adaptive spectrum sensing for cognitive radio based on multi-objective genetic optimisation. IEEE Electron. Lett. 2013, 49, 1099-1101.

6. Liu, N.H.; Chiang, C.Y.; Hsu, H.M. Improving Driver Alertness through Music Selection Using a Mobile EEG to Detect Brainwaves. Sensors 2013, 13, 8199-8221.

7. Li, S.G.; Sharkh, S.M.; Walsh, F.C.; Zhang, C.N. Energy and Battery Management of a Plug-In Series Hybrid Electric Vehicle Using Fuzzy Logic. IEEE Trans. Veh. Technol. 2011, 60, 3571-3585.

8. Zhang, Y.; Liu, H.; Guo, Q. Varying-Domain Optimal Management Strategy for Parallel Hybrid Electric Vehicles. IEEE Trans. Veh. Technol. 2014, 63, 603-616.

9. Kubb, A.E.; Jiang, K. A Comprehensive Study on Technologies of Tyre Monitoring Systems and Possible Energy Solutions. Sensors 2014, 14, 10306-10345.

10. Kawecki, L.; Niewierowicz, T. Hybrid Genetic Algorithm to Solve the Two Point Boundary Value Problem in the Optimal Control of Induction Motors. IEEE Lat. Am. Trans. 2014, 12, 176-181.

11. Roy, H.K.; McGordon, A.; Jennings, P.A. A Generalized Powertrain Design Optimization Methodology to Reduce Fuel Economy Variability in Hybrid Electric Vehicles. IEEE Trans. Veh. Technol. 2014, 63, 1055-1070.

12. Lao, S.I.; Choy, K.L.; Ho, G.T.S.; Yam, C.M.R.; Tsim, Y.C.; Poon, T.C. Achieving quality assurance functionality in the food industry using a hybrid case-based reasoning and fuzzy logic approach. ELSEVIER Expert Syst. Appl. 2012, 39, 5251-5261.

13. Silva, L.W.T.; Barros, V.F.; Silva, S.G. Genetic Algorithm with Maximum-Minimum Crossover (GA-MMC) Applied in Optimization of Radiation Pattern Control of Phased-Array Radars for Rocket Tracking Systems. Sensors 2014, 14, 15113-15141.

14. Ullah, S.; Groen, T.A.; Schlerf, M.; Skidmore, A.K.; Nieuwenhuis, W.; Vaiphasa, C. Using a Genetic Algorithm as an Optimal Band Selector in the Mid and Thermal Infrared $(2.5-14 \mu \mathrm{m})$ to Discriminate Vegetation Species. Sensors 2012, 12, 8755-8769.

15. Toyota is Global Hybrid Leader with Sales of 7 Million. Available online: http://www.toyotanewsroom.com/releases/toyota+global+hybrid+leader+sales $+7 \mathrm{~mm} . \mathrm{htm}$ (accessed on 14 October 2014).

16. Toyota Prius c Specification. Available online: http://www.priusc.com.hk/spec.pdf (accessed on 11 June 2014).

17. Greenhouse Gas Emissions from a Typical Passenger Vehicle. Available online: http://www.epa.gov/otaq/climate/documents/420f11041.pdf (accessed on 21 August 2014).

(C) 2015 by the authors; licensee MDPI, Basel, Switzerland. This article is an open access article distributed under the terms and conditions of the Creative Commons Attribution license (http://creativecommons.org/licenses/by/4.0/). 